\title{
Verzeichnis der Partisanenfotografen
}

Die Namen vieler Partisanenfotografen sind unbekannt und viele werden es auch bleiben An dieser Stelle sind die Namen aller Fotografen aufgelistet, die im Zuge der Untersuchungen zum vorliegenden Buch erfasst werden konnten. Mit dem Verzeichnis soll nochmals das Ausmaß der fotografischen Produktion innerhalb der Partisanenbewegung aufgezeigt und eine Grundlage für weitere Forschungen geschaffen werden.

Abramović, (Miloš) Slavka (geb. 1925)

Geboren in Beli Manastir. Ab Oktober 1944 Kämpfer der Ersten Proletarischen Brigade. Mitglied der Stabseinheit des Ersten Bataillons.

Afrić, Vjekoslav (1906-1980)

Der Schauspieler des Kroatischen Nationaltheaters in Zagreb schloss sich 1942 den Partisanen an, wurde Mitglied des Theaters der Volksbefreiung und Fotograf der Partisanen.

Aković, (Velije) Ismet (1920-1945)

Fotograf aus Gračanica in Bosnien und Herzegowina. Schloss sich der Volksbefreiungsarmee am 18. Februar 1945 an. Er fiel am 15. April 1945 auf dem Berg Romanija.

Andjelić, Ljubo (gest. 1943)

Fotografierte während der Fünften Offensive, bei der seine Filme jedoch vernichtet wurden. Am 9. Juni nahm er den verwundeten Josip Broz Tito und zuvor Vladimir Nazor bei Vučevo auf. Andjelić fiel 1943 in den Bergen von Ljubin Grob bei Tjentište.

\section{Baić, Dušan}

Fotograf des Vierten Korps der Volksbefreiungsarmee Jugoslawiens bzw. des Ersten Korps der Volksbefreiungsarmee Kroatiens.

Bajić, Krsto (1919-1944)

Volksheld und Fotograf. Politkommissar des Ersten Bataillons der Ersten Proletarischen Brigade. Er fiel am 23. August 1944 bei den Kämpfen gegen die Tschetniks auf dem Berg Zlatibor.

\section{Bakarić, Vladimir (1912-1983)}

Vor dem Krieg Sekretär des Verbands der Kommunistischen Jugend Jugoslawiens an der Universität Zagreb, weshalb er zu einer Gefängnisstrafe von sechs Monaten in Belgrad verurteilt wurde. Während des Krieges schloss er sich nach illegaler Tätigkeit in der Widerstandsbewegung den Partisanen an und wurde Politkommissar des Hauptstabs Kroatiens. Bakarić war einer der Gründer des Antifaschistischen Landesrats der Volksbefreiung Kroatiens und übernahm 1944 von Andrija Hebrang die Funktion des Sekretärs des Zentralkomitees der Kommunistischen Partei Kroatiens. Nach dem Krieg Ernennung zum ersten Ministerpräsidenten der Regierung 
der Republik Kroatien und Aufstieg zu einem der mächtigsten Politiker. Die gesamte Zeit als Fotograf der Partisanen tätig.

Baruh, Jakov (geb. 1908)

Geboren am 11. September 1908 in Banja Luka, wo er als Fotograf arbeitete. Nach der Ausrufung des Unabhängigen Staates Kroatien wurde sein Fotostudio Rekord geschlossen. Er floh nach Split, wo ihn die italienischen Besatzungsmächte fassten und in das Lager Vela Luka auf der Insel Korčula brachten. 1942 schloss er sich der Volksbefreiungsarmee an, zunächst als Kämpfer in der Korčula-Einheit und folgend in verschiedenen dalmatinischen Partisaneneinheiten.

Baruh, Moric (geb. 1911)

Geboren in Sarajevo. Als Fotograf in Banja Luka tätig. Nach der Ausrufung des Unabhängigen Staates Kroatien floh er nach Split, wo er 1943 der Volksbefreiungsarmee beitrat. Er diente in verschiedenen dalmatinischen Partisaneneinheiten.

\section{Batinić, Andjelko}

Fotograf des Vierten Bataillons der Ersten Dalmatinischen Brigade und Mitglied der Fotosektion des Regionalen Volksbefreiungsrates für Dalmatien.

\section{Bavec, Marija (1904-1985)}

Geborene Lovšin. Einzige slowenische Partisanenfotografin und Ehefrau des Fotografen Vinko Bavec. Besonders interessant sind ihre Fotografien der Partisaninnen und der eigenen Familie während des Krieges, die heute im Museum für Neuere Geschichte in Ljubljana verwahrt werden.

Bavec, Vinko (1899-1969)

Kaufmann und Soldat im Ersten Weltkrieg. Er war einer der erfolgreichsten Verleger von Ansichtskarten und im Zweiten Weltkrieg als Partisanenfotograf tätig.

\section{Benčić, Stevan}

Vor dem Krieg Fotograf der Tageszeitung Politika in Belgrad. 1930 zog er auf die Insel Hvar, baute dort ein florierendes Fotogeschäft auf und war ab 1943 Mitglied der Partisanen.

Beran, Jan (1927-1993)

Fotograf der Partisanen und bekannter Nachkriegsfotograf, Regisseur, Dramaturg, Szenarist und Übersetzer.

\section{Bervar, Ciril}

Fotograf.

\section{Berzoli, Lauro}

Fotograf des Dritten Bataillons der Sechsten Proletarischen Division.

Bibić, Nikola (1923-2001)

Fotograf und Kämpfer der 13. Proletarischen Brigade. Schöpfer von Fotografien des Ersten Proletarischen Bataillons Kroatiens. Er organisierte in den Partisaneneinheiten Kurse für Amateurfotografen und war nach dem Krieg als Fotograf der Tageszeitung Borba äußerst erfolgreich.

Bitenc, Jože (geb. 1893)

Eisenbahner, der sich 1941 der Kranj-Kompanie anschloss und bei den Partisanen seine ersten Fotografien aufnahm.

\section{Bojčević, Pavle}

Mitglied der Zweiten Dalmatinischen Proletarischen Brigade, Partisanenfotograf und Radiotelegrafist.

\section{Borovčanin, Grujo}

In Ostserbien bekannt unter dem Spitznamen »doktor aktivista« (Doktor-Aktivist) und »seoski fotograf» (Dorffotograf).

Bračič, Mirko (1915-1943)

Bekannt auch unter seinem Partisanendecknamen Miran Bradač. Partisanenkommandant, Politkommissar und Volksheld, der unter 
anderem Kommandant der Laastal-Einheit, der operativen Alpen-Zone und der 14. Slowenischen Division war. Schöpfer zahlreicher Fotografien aus dem Partisanenleben. Bračić fiel bei der Befreiung von Kočevje, versehentlich getroffen von Schüssen, die Franc Bobnar-Gedžo abgefeuert hatte.

Brelih, Miloš (1916-2002)

Partisanenfotograf und Ingenieur. Einer der Gründer von Kričač, einer illegalen Rundfunkstation, die von November 1941 bis April 1942 im besetzten Ljubljana aktiv war. Später Leiter der Abteilung für Funktelegrafie des Hauptstabs der slowenischen Partisanen. Nach dem Krieg, im Jahr 1945, wurde er Direktor der ehemals deutschen Fabrik für Flugzeugteile in Maribor, die er zur ersten slowenischen Automobilfabrik namens TAM

umwandelte.

Brenk, France (geb. 1912)

Er war im Agitprop des Rates der Volksbefreiung Sloweniens tätig, organisierte Fotokurse und leitete die Fotosektion.

Brvar, Ciril (geb. 1920)

Mitglied der Fotosektion der Vierten operativen Zone. Er arbeitete eng mit Jože Petek zusammen, der die Sektion in dieser Zeit leitete.

Bulatović, Raca (geb. 1923)

Geboren in Djakovica. Ab 1941 Kämpfer in der Volksbefreiungsarmee. Aus den montenegrinischen Verbänden wurde er 1944 in die Zweite Proletarische Brigade abkommandiert. Er war Politkommissar des Krankenhauses und der Aufklärungskompanie. Gleichzeitig fotografierte er während des gesamten Krieges.

Čaldarević, Mladen (1916-2010)

Geboren in Bijeljina. Studierte in Zagreb Pädagogik, Philosophie, Englisch und Psychologie und diplomierte nach dem Krieg in Belgrad. Als namhafter linker Intellektueller entzog er sich der
Verfolgung durch die Ustascha, indem er sich am 10. April 1941 den Partisanen in Jabukovac bei Petrinja anschloss. Er fotografierte die Kämpfe am Fluss Sutjeska. Seine Fotosammlung befindet sich heute im Kroatischen Staatsarchiv. Nach der Spezialisierung im Bereich der Soziologie unterrichtete er an Hochschulen in Zagreb, Belgrad und Sarajevo sowie in den USA an den Universitäten Columbia, Stanford und Berkeley.

\section{Čanak, Ratko}

Fotograf.

\section{Čarkić, Mišo}

Fotograf und Mitglied des Verbands der Kommunistischen Jugend Jugoslawiens.

Cerar, France (geb. 1912)

Er war Gefangener im italienischen Lager auf der Insel Rab und wurde nach der Kapitulation Italiens Kommissar der Artilleriebrigade der 14. Division. Als Verwundeter fotografierte er das Partisanenkrankenhaus auf dem Höhenzug Kočevski Rog. Zudem war er Mitglied der Fotosektion des Rats der Volksbefreiung Sloweniens und Mitglied der Fotosektion des Hauptstabs Sloweniens.

\section{Cerjan, Stjepan-Puba}

Zagreber Untergrundkämpfer aus Trnje, Mitglied der Kommunistischen Jugend und Fotograf, der 1945 bei Mraclin verwundet wurde.

Ciglič, Marjan (geb. 1924)

Geboren in Kranj. Als Mitglied der Befreiungsfront half er bei der Erstellung von Fotografien, auf denen Verbrechen der deutschen Wehrmacht festgehalten wurden. Später war er Kämpfer und Kommissar des Ersten Bataillons der Prešern-Brigade, Maschinengewehrschütze und Fotograf. In der Brigade war er für Agitation und Propaganda zuständig. 
Čitaković, Sreten (geb. 1917)

Partisanenkämpfer in der Kolubara-Kompanie.

Er fotografierte Soja Stanišić, eine der ersten Partisaninnen, und schuf zu Beginn des Krieges Porträts von Ivo Lola Ribar und Aleksandar Ranković.

\section{Crnelić, Milan}

Mitglied des Fotodienstes des Regionalen Volksbefreiungsrates für Slawonien.

\section{Ćurćić, Milica}

Fotografin.

Cvrtila, Franjo (geb. 1927)

Trat der Partisanenbewegung im Alter von 16 Jahren bei. Mitglied der 16. Jugendbrigade Joža Vlahović. Lebt heute in Zagreb.

Damnjanović, Živorad (gest. 1943)

Fotograf und Mitglied des Verbands der Kommunistischen Jugend Jugoslawiens. Er fiel 1943.

Deković, Ivan (1921-1944)

Fotograf aus Rogoznica und Mitglied der Widerstandsbewegung. Er wurde in Stupina hingerichtet.

\section{Dolački, Josip}

Leiter der Fotosektion in Pokupsko. Seine Fotografien aus Zagreb gelangten über Verbindungsmänner nach Pokuplje und Moslavina und wurden von dort aus verbreitet.

\section{Dragila, Pero}

Mitglied des Fotodienstes des Regionalen Volksbefreiungsrates für Slawonien. Dragila fotografierte die Befreiung von Zagreb. Nach dem Zweiten Weltkrieg und der Resolution des Informationsbüros emigrierte er nach Prag.

Elijas, (Rudolf) Tauber (1920-1944)

Fotograf aus Sarajevo. Nach der Ausrufung des Unabhängigen Staates Kroatien floh er in die italienische Besatzungszone. In Dalmatien wurde er im Lager auf der Insel Rab interniert und schloss sich von dort aus am 9. September 1943 der Partisanenbewegung an. Ende 1944 fiel er in der Nähe von Banijski Jabukovac.

Ercegović, (Ivan) Josip (geb. 1907)

Fotograf. In Zagreb erlernte er die Holzschnitzerei und wirkte in der gewerkschaftlichen Arbeiterbewegung. Nach Ausbruch des Zweiten Weltkrieges zog er sich in den Untergrund zurück und trat 1943 der Partisaneneinheit im Mosor-Gebirge bei. In der Folge war er Mitglied des Ersten Montenegrinischen Bataillons der Ersten Proletarischen Stoßbrigade, wo man ihn mit seinen beiden Fotoapparaten in den Stab der Ersten Proletarischen Division abkommandierte. Er bekleidete zugleich den Posten des Kurierdienstleiters. Man verhaftete ihn einige Male, doch gelang es ihm immer, zuvor vertrauliches Material zu vernichten.

\section{Faneli, Mauricije Mario}

Fotografierte die Befreiung von Belgrad und Zagreb. Alle Filme aus der Kriegszeit entwickelte er erst nach seiner Rückkehr nach Zagreb.

Fischer, Hari (geb. 1918)

Geboren in Wien, arbeitete er als Fotograf in Dubrovnik. Unter italienischer Besatzung floh er wie die meisten Juden vor dem Terror der Ustascha. Er wurde im Lager Kupare unweit von Dubrovnik und später im Lager auf der Insel Rab interniert. 1943 schloss er sich von dort aus der Partisanenbewegung als Kämpfer der Siebten Banija-Division an, um später Fotograf in der Fotosektion des Siebten Korps zu werden.

Fischer Ribarić, Hugo (geb. 1903)

Nach der Ausrufung des Unabhängigen Staates Kroatien flüchtete er in die italienische Besatzungszone, wo er jedoch als Jude 1942 in das Konzentrationslager Kraljevica und später in das Lager auf der Insel Rab gebracht wurde. Nach der Kapitulation Italiens und der Befreiung des Lagers im Jahr 1943 trat er der Volksbefreiungsarmee bei und wurde einer der Begründer der Fotosektion des Landesrates der Volksbefreiung Kroatiens. 
Gattin, Živko (1923-2018)

Einziger Fotograf, der während des Zweiten

Weltkriegs Farbfotografien in Kroatien aufnahm, und einer der Gründer des dalmatinischen Fotodienstes Slobodna Dalmacija (Freies Dalmatien). Nach dem Krieg war er kurzzeitig Leiter des Zentrums für Fotodokumentation innerhalb der Presseabteilung des Parlaments der Republik Kroatien, daraufhin widmete er sich jedoch gänzlich seiner Tätigkeit als Landwirt.

Gavrić, Tiosav (geb. 1920)

Geboren im Dorf Degurić bei Valjevo. Kaufmann und während des Krieges Fotograf der Dritten Kompanie des Ersten Bataillons.

Goldstein, Emil (geb. 1920)

In Sarajevo geborener Berufsfotograf. Verwundet während der Sechsten feindlichen Offensive, woraufhin man ihn in die Agitprop-Abteilung der Neunten Dalmatinischen Division und des Achten Korps abkommandierte.

\section{Grbin, Ljubomir}

Nahm Fotografien in El Shatt, Ägypten, auf.

Gregorić, Pavle (1892-1989)

In der Vorkriegszeit Kommunist und Mitglied des Zentralkomitees der Kommunistischen Partei Kroatiens. Einer der Gründer des Landesrats der Volksbefreiung Kroatiens sowie Abgeordneter in der Ersten und Zweiten Sitzung des Antifaschistischen Rats der Volksbefreiung Jugoslawiens. Ein Teil seines fotografischen Werks wird im Kroatischen Staatsarchiv verwahrt und ist eingeschränkt für die Öffentlichkeit zugängig.

Grgić, Ernest (geb. 1913)

Geboren in Sarajevo. Vor dem Krieg Fotograf in Zagreb und ab 1943 Mitglied der Kommunistischen Partei Jugoslawiens. Im Spanischen Bürgerkrieg kämpfte er in der Einheit Divizionario der 45. Division. Er stand dem Politkommissar Blagoje Parović nahe. Als Unteroffizier kehrte er 1938 aus Spanien zurück. Im Volksbefreiungskrieg war er für die Radiotelegrafie im Stab der Ersten Proletarischen Division zuständig und wurde bei Kämpfen für die Befreiung von Zvornik verwundet. Später folgte er dem Ruf von Vili Šimunov-Barba, sich der Presseagentur TANJUG anzuschließen und mit ihm zusammen ein Fotoarchiv zu erstellen, das Ivo Lola Ribar nach Kairo mitnehmen sollte. Verheiratet mit Dina Zlata Grgić.

\section{Gvozdenović, Jovica}

Fotograf und Mitglied der 31. Serbischen Stoßbrigade der Volksbefreiungsarmee.

\section{Hreljanović, Viktor (1923-1997)}

Aus Rijeka stammender Fotograf, der die Befreiung seiner Geburtsstadt fotografierte.

\section{Ilić, Milenko}

Fotograf aus Kosjerić.

\section{Ingolič, Anton (1907-1992)}

Aus Slowenien stammend, wurde er zu Beginn des Krieges nach Serbien vertrieben. Seinen Aufenthalt in Serbien hielt er auf circa zweihundert Fotografien fest und dokumentierte das Leben in den Lagern in Paraćin und Zaječar mit seinem Fotoapparat, den er heimlich ins Lager schmuggelte. In Ćuprija gelang es ihm, die Hinrichtung der Widerstandskämpfer am Galgen im Stadtzentrum zu fotografieren. Berühmter Schriftsteller.

Ivančić, Stane (geb. 1911)

Geboren in Bovec. Der Volksbefreiungsarmee trat er am 2. Februar 1943 bei.

\section{Ivanovski, Ivan}

Fotolaborant und Zugführer der 42. Fliegerdivision.

Iveković, Mladen (1903-1970)

Promovierte 1928 und arbeitete als Jurist. Vor dem Krieg Mitglied der Kommunistischen Partei Jugoslawiens, inhaftiert 1936 und 1938. Mitglied des Agitprop und des Zentralkomitees der Kommunistischen Partei Kroatiens. Redakteur 
zahlreicher Zeitschriften und Gründer der Zeitschrift Pregled. Gemeinsam mit Andrija Hebrang kam er durch Gefangenenaustausch aus dem Lager Jasenovac frei. Mitglied des Exekutivrats des Antifaschistischen Rats der Volksbefreiung Jugoslawiens, Chefredakteur der Zeitschrift Vjesnik und Leiter der Propagandaabteilung des Antifaschistischen Landesrats der Volksbefreiung Kroatiens sowie einer der bedeutendsten Akteure beim Ersten Kongress der Kulturschaffenden Kroatiens in Topusko (25-27. Juni 1944). Fotografierte während des Krieges. Sein Nachlass befindet sich heute im Kroatischen Staatsarchiv, seine Fotografien werden im Kroatischen Historischen Museum verwahrt.

Jakac, Božidar (1899-1989)

Geboren in Novo Mesto, Slowenien. Maler und Fotograf, Pädagoge und Dokumentarist, der 1943 den Partisanen folgte. Er schuf zahlreiche Grafiken sowie Fotografien und war einer der zentralen Organisatoren bei der Gründung der Akademie der bildenden Künste und des Designs in Ljubljana.

\section{Jakovljević, Vladeta}

Fotolaborant und junger Zugführer der 42. Fliegerdivision.

Janać, (Jožef) Petar (geb. 1927)

Fotograf aus Bačka Palanka und Mitglied der Jugendkompanie.

Jančić, (Radoslav) Angelina (geb. 1926)

Fototechnikerin.

Janković, (Krsta) Aleksandar (geb. 1909)

Geboren in Drenovac bei Kragujevac. In der Dritten Krajina-Brigade wirkte er ab Oktober 1944 als Fotograf.

\section{Janković, Simon}

Mitglied der Zehnten Herzegowina-Brigade. Mit seinem eigenen Fotoapparat schuf er Aufnahmen während des Krieges.

\section{Jelič, Peter}

Fotograf.

Jeraj, Jožica (geb. 1925)

Gesellin im Fotoatelier, in dem auch der Fotograf Miloš Trobec arbeitete. Mit Trobec fertigte sie Fotografien für die Untergrundkämpfer und war ab 1944 in der Fotosektion des Hauptstab Sloweniens tätig.

Jesenovec, Sandi (geb. 1926)

Geboren in Škofja Loka, wo er im Fotostudio Šelhaus arbeitete. Gemeinsam mit Edi Šelhaus schuf er zu Beginn des Zweiten Weltkriegs Fotos für die Widerstandsbewegung. Am 3. August 1943 schloss er sich den Partisanen an, bei denen er zunächst als Kurier fungierte und ab dem 9. Juni 1944 dem Stab des Neunten Korps zugeteilt wurde, wo er die Organisation des Fotolabors in der Sektion für Geodäsie übernahm. Er machte Aufnahmen im Format $9 \times 12 \mathrm{~cm}$. Das Museum für Neuere Geschichte in Ljubljana verwahrt heute 2.506 Negative aus seiner Hand. Er fotografierte viele Partisanen, aber auch die Befreiung der Städte Triest und Gorica.

Jokić, (Jovan) Rade (1907-1992)

Geboren in Valjevo, aus einer Arbeiterfamilie stammend. Mitglied der Arbeiterbewegung und der Kommunistischen Partei Jugoslawiens ab 1937. Seine Schwägerin war die Ehefrau des Malers Djordje Andrejević Kun. Im Krieg gehörte er der Valjevo-Kompanie an und geriet 1942 in Gefangenschaft. Im Juni 1944 schloss er sich nach der Haft erneut den Partisanen an.

\section{Jovanović, Jovan}

Leiter des Fotodienstes, Leitungsassistent des Nachrichtendienstes und Unterleutnant der 42. Fliegerdivision.

Jovanović, (Jovo) Drago (1900-1943)

Geboren in Priboj bei Lopare, erlernte er nach Beendigung der Volksschule das Fotografenhandwerk und eröffnete das Fotogeschäft Foto Drago. Während des Zweiten Weltkriegs arbeitete er mit 
der Untergrundbewegung zusammen und entwickelte Filme der Partisanen, die ihm aus dem Majevica-Gebirge übermittelt wurden. In seinem Fotostudio hielt man illegale Sitzungen ab. Im November 1943 verließ er gemeinsam mit den Einheiten der Volksbefreiungsarmee Tuzla und fiel im selben Jahr in der Nähe von Kalesije.

\section{Kabić, Danilo}

Fotoreporter der Nachrichtenagentur TANJUG und einer der bekanntesten Fotografen.

\section{Kalajdžić, Mustafa}

Fotograf der Fotosektion der 13. Herzegowina-Stoßbrigade in der Propagandaabteilung des Brigadestabs.

\section{Kapor, Čedo (1914-2004)}

Geboren in Trebinje, kämpfte er im Spanischen Bürgerkrieg. Kommissar der Herzegowina-Brigade und Fotograf des Ersten HerzegowinischMontenegrinischen Stoßbataillons. Zudem war er Sekretär des Regionalen Volksrates für die Herzegowina sowie Ausbilder des Operativen Stabs für die Herzegowina.

Kenelić, (Stevana) Dušan (geb. 1926)

Geboren in Bokšić Lug bei Našice. Fotograf im Stab der Dritten Proletarischen Krajina-Brigade.

Klemenčič, Dore (1911-1988)

Maler, Mitglied der Šercer-Brigade, Leiter der Abteilung für grafische Gestaltung, Literatur und Fotografie im Neunten Korps. Darüber hinaus war er in der Bildungsabteilung im Rat der Volksbefreiung Sloweniens in Črnomelj aktiv.

\section{Klis, Klaus Vladimir}

Slowenischer Partisanenfotograf. 1945 hielt er die Folgen der Bombardierung der Stadt Zadar und am 9. Mai 1945 die Befreiung von Ljubljana fest.

Knežić, Luka (1912-1943)

Vor dem Krieg Mitglied der Arbeiterbewegung, ab 1941 Mitglied der Kommunistischen Partei
Jugoslawiens und Fotograf. Ab Juni 1941 diente er im Mostar-Bataillon und fiel in Šćit, unweit der Ortschaft Prozor in Bosnien und Herzegowina.

Kohn, Elvira (1904-2003)

Geboren in Rijeka. Vor dem Krieg arbeitete sie als Fotografin in Dubrovnik. Sie schmuggelte ihre Kamera der Marke Leica in das Konzentrationslager auf der Insel Rab und fertigte auch Aufnahmen während ihrer Gefangenschaft.

\section{Kolobratar, Djuro}

Fotograf aus Bor. Mitglied der Zehnten Brigade der 23. Division der jugoslawischen Volksbefreiungsarmee.

\section{Konjhodžić, Mahmud (1905-1979)}

Geboren in Ljubuško in Bosnien und Herzegowina. Jurastudium in Belgrad und Zagreb, wo er 1931 seinen Hochschulabschluss erlangte. Anschließend arbeitete er als Korrespondent für die Zeitung Politika. Zu Beginn des Zweiten Weltkriegs agierte er als Untergrundkämpfer in Zagreb, in der Folgezeit war er als Journalist und Fotograf in der Propagandaabteilung des Antifaschistischen Landesrats der Volksbefreiung Kroatiens, der Redaktion der Zeitung Vjesnik und der ersten Redaktion der Nachrichtenagentur TANJUG tätig. Autor zahlreicher Reportagen und Texte.

\section{Kopinič, Dare}

Fotograf des Rats der Volksbefreiung Sloweniens. Die Fotosektion dieses Rats war in Bordons Haus in Črnomelj untergebracht, wo mit der Einrichtung des Fotoarchivs der slowenischen Partisanen begonnen wurde.

\section{Koprivec, Ignac (1907-1980)}

Leiter der Fotosektion des Hauptstabs für Slowenien und Redakteur der Zeitung Slovenski Partizan.

Korčagin, Mića (geb. 1922)

In Smederevska Palanka geborener Fotograf. 


\section{Koren, Djurdja}

Fotografin aus Velika Gorica, die nach ihrer Heirat den Namen Šipušić annahm.

\section{Korsun, $\mathbf{N}$}

Fotograf.

\section{Kos, Alfred}

Mitglied der Fotosektion des Rats der Volksbefreiung Sloweniens.

Kostić, (Matija) Vlado (geb. 1922)

Geboren in Ličje bei Gadžin Han, Serbien. Ab November 1944 Kämpfer des Ersten Bataillons der Ersten Proletarischen Brigade.

Kotnik, Jože (geb. 1914)

Fotografierte auf dem befreiten Territorium bei Kočevje.

\section{Kovač, Voja}

Leiter der Propagandaabteilung der Zweiten Brigade der 25. Serbischen Division.

\section{Krajačić, Ivan-Stevo (1906-1986)}

Einer der bedeutendsten Geheimagenten, der während des gesamten Krieges fotografierte.

All seine Fotografien und sein Fotoapparat gingen beim Flugzeugabsturz bei Čemernica verloren, als auch Randolph Churchill, der Sohn von Winston Churchill, verwundet wurde.

\section{Krnjajić, Petar}

Fotografierte im Mai 1942 die regionale Parteikonferenz für Banija in Ljeskovac. Gleichzeitig fotografierte er kontinuierlich für die feindlichen Streitkräfte. Später wurde er wegen des Niederbrennens des Dorfes Prekop verurteilt und durch Erschießung hingerichtet.

\section{Krajina, Rudi}

Fotograf.

\section{Kramar, Viktor}

Slowenischer Fotograf und Untergrundkämpfer.
Križan, Janko (geb. 1918)

Geboren im Dorf Boljevci bei Zemun, wo er als Fotograf arbeitete. Zugführer in der Volksbefreiungsarmee.

\section{Kržišnik, Zdravko (geb. 1921)}

In der Fotosektion des Hauptstabs für die Entwicklung der Negative und die Ordnung des Fotoarchivs zuständig. Am 28. März 1943 fotografierte ihn France Cerar im Fotolabor in Črnomelj.

\section{Latinović, Milan}

Fotograf der Stabseinheit der Ersten Proletarischen Brigade.

Lekić, (Aksentija) Radisav (1921-1942)

Geboren in Mrčajevci. Als Mitglied des Ljubić-

Bataillons geriet er in Gefangenschaft und wurde am 8. Dezember 1942 von bulgarischen Streitkräften durch Erschießung hingerichtet.

Lenardič, Stane-Žan (1918-2008)

Begann bereits mit 14 Jahren zu fotografieren. Bei den Partisanen wirkte er als Kriegsberichterstatter und Fotograf. Er nahm furchtlos aus nächster Nähe die Angriffe der Schlandroff-Brigade auf. Später organisierte er Filmaktivitäten und war Korrespondent der Nachrichtenagentur TANJUG für die slowenische Adria.

\section{Lilik, Miroslav}

Fotograf. Er nahm die Kriegsgefangenen auf dem Bleiburger Feld auf.

\section{Lipar, Ivan-Iztok}

Fotograf.

\section{Lisac, Ivica}

Aus Sarajevo stammender Fotograf und Untergrundkämpfer.

\section{Lukateli, Anton (geb. 1916)}

Maler und Fotograf während der Schlacht an der Sutjeska. Ab 1941 im Volksbefreiungskampf aktiv. 
Magajna, Mario (1916-2007)

Geboren in Triest. Mitglied der Befreiungsfront. Im Triester Krankenhaus fertigte er Aufnahmen mit Hilfe von Röntgenplatten, zudem fotografierte er die Kämpfe um die Befreiung von Triest.

\section{Manjiček, Franjo}

Landwirt. Wegen seiner Invalidität vom Militärdienst befreit. Er konnte sich frei bewegen und dokumentierte fotografisch die Ereignisse während des Krieges. Die Ustascha nahmen ihn gefangen und brachten ihn ins Konzentrationslager Jasenovac, wo er 1944 ermordet wurde.

Marenčić, Janez (1914-2007)

Jurist und während des Krieges Mitglied des Neunten Slowenischen Korps. Die meisten seiner Fotografien schuf er in der Küstenlandbrigade.

\section{Marinček, Ivan}

Geboren in Nova Vas in der Nähe von Ptuj. Während des Krieges Radiotechniker des Hauptstabs für Slowenien. Er fertigte Aufnahmen des Kongresses der Antifaschistischen Frauenfront in Ljubljana, war Meister der Schwarzweißfotografie und einer der Pioniere des slowenischen Films.

\section{Marjanović, Risto (1885-1969)}

Im Ersten Weltkrieg Kriegsberichterstatter und aus heutiger Sicht Begründer der serbischen Fotoreportage. Er lehnte es ab, für die serbische Marionettenregierung zu arbeiten. Während des Zweiten Weltkriegs gelangen ihm heimliche Aufnahmen, im Jahr 1944 dokumentierte er die Befreiung Belgrads.

\section{Masterl, Marjan (geb. 1917)}

Fotografierte während der Okkupation von Škofija Loka. In der Folge trat er der Gorenje-Einheit bei, wo er als Offizier des Nachrichtendienstes und als Fotograf arbeitete. Seine außerordentlich interessanten Fotografien entstanden anlässlich des ersten Skiwettbewerbs der Partisanen am 21. Januar 1945.

\section{Matašin, Miro}

In der Vorkriegszeit Mitglied des Verbands der Kommunistischen Jugend Jugoslawiens. Leiter der Fotosektion der Zwölften Stoßdivision und Leiter der Fotosektion des Regionalen Volksbefreiungsrats Slawoniens.

\section{Mate, Jelič}

Leiter der Fotosektion des Neunten Korps.

Mažar, Drago (1918-1991)

Volksheld. Bevor er sich der Partisanenbewegung anschloss, stand er an der Spitze einer Diversanten-Gruppe. Bei den Partisanen war er Kommandant der Ersten Kompanie für die Bosnische Krajina und später Kommandant der Sechsten Partisaneneinheit sowie Offizier des Nachrichtendienstes im Operativen Stab der Bosnischen Krajina. Er verlor im Krieg drei Brüder und seine Mutter.

\section{Mehobej, Stevo}

Fotografierte den Einzug der Partisanen in Zagreb.

\section{Mešterović, Djura}

Fotograf.

Mičić, Milorad (geb. 1911)

Geboren in Belgrad. Mičić fiel bei Lovas als Mitglied der Vierten Serbischen Stoßbrigade.

Milčinski, Janez (1913-1993)

Jurist und Arzt. Während des Zweiten Weltkriegs arbeitete er als Arzt und nahm viele künstlerisch hochwertige Fotografien auf. Er war einer der Gründer des Sanitätsdienstes der Partisanen sowie Arzt im Partisanenkrankenhaus und der Entbindungsstation Spodnji Hrastnik auf dem Höhenzug Kočevski Rog, woher die erhalten gebliebenen Fotografien stammen.

\section{Milković, Simo}

Illegal tätiger Fotograf aus Rijeka.

\section{Mihić, Mate}

Kommissar und Fotograf. 


\section{Mitrović, Mitar}

Fotograf.

Mlekuž, Riko (geb. 1910)

Vor Ausbruch des Zweiten Weltkriegs arbeitete er im Fotoatelier Purač in Murska Sobota, später im Studio Kramarič in Ljubljana. Als Mitorganisator des Attentats auf Benito Mussolini wurde er verhaftet und nach Rom geschickt, doch gelang es ihm, aus dem Zug zu entkommen. In Celje wirkte er kurzzeitig im Studio Pelikan und floh anschließend zu den Partisanen, wo er als Fotograf der 14. Division tätig war.

Mosinger, Franjo (1899-1956)

Einer der bedeutendsten kroatischen Fotografen der Zwischenkriegszeit. Nach einem Architekturstudium in Wien kehrte er 1918 nach Zagreb zurück und übernahm das Atelier seines Vaters in Ilica - das erste kroatische Institut für Fotokunst. Später verlegte er sein Atelier in den Zagreber Stadtteil Dolac und eröffnete 1935 ein Atelier in der Knez-Mihailova-Straße in Belgrad. Seine Reise zu den Partisanen am 11. Mai 1943 dokumentierte er in Form eines Fototagebuchs unter dem Namen Slobodan Antunović. Er wirkte im Agitprop des Zentralkomitees der Kommunistischen Partei Kroatiens und gründete eine Fotosektion in Otočac. Ein Jahr später wurde er schwer verwundet und über die Insel Vis ins Krankenhaus nach Bari transportiert. Während dieser Zeit schuf er die politischen Karikaturen Bolesnici sobe br. 22 (Patienten aus Zi. 22) und Medicinari u Grumu (Mediziner in Grum).

Munjas, Petar (1922-1978)

Geboren im Dorf Gojak. Ab 1933 erlernte er in Belgrad das Fotografenhandwerk, verließ nach Schwierigkeiten jedoch seinen Meister und vollendete seine Lehre in Smederevska Palanka, wo er sich der gewerkschaftlichen Arbeiterbewegung anschloss.

\section{Nedeljković, Konstantin}

Berufsfotograf und Sympathisant der Kommunistischen Partei Jugoslawiens aus Leskovac. 1941 wurde er verhaftet und im Konzentrationslager Banjica hingerichtet.

\section{Nešković, Bora}

Fotograf.

\section{Nikolić, Dragan}

Fotograf.

\section{Nikolić, (Save) Zdravko (geb. 1920)}

In Ljubinić bei Obrenovac in Serbien geborener Kaufmann. Ab 1944 im Volksbefreiungskrieg als Fotograf tätig. Fotograf der Ersten Brigade der Sechsten Proletarischen Division.

\section{Nikoliš, Branko}

Aus Sjeničak stammend, war er vor dem Krieg als Kaufmann tätig. Er fotografierte Piloten der Alliierten in der kroatischen Ortschaft Radjenovac unweit von Novska und die Kolonnen ziviler Geflüchteter in Vrgin Most.

\section{Nisim, Baruh (1915-1943)}

Geboren in Banja Luka, in Belgrad als Fotograf tätig. Mitglied der Kommunistischen Partei Jugoslawiens ab Juli 1943. Als Kämpfer des Sechsten Belgrader Bataillons der Ersten Proletarischen Brigade fiel er im November 1943 bei den Kämpfen um die Befreiung von Travnik.

\section{Oblak, Matej}

Fotograf in der Fotowerkstatt Vipava.

Orlić, Zvonimir (1910-1942)

Geboren in Pula. Berufsfotograf und ab April 1942 Mitglied des Mostar-Bataillons. Im Juli 1942 fiel er in Ostrožac unweit von Konjic.

Orović, Savo (1888-1974)

Offizier der montenegrinischen Armee im Ersten Weltkrieg und Offizier im Königreich der Serben, Kroaten und Slowenen, der den Rang eines Obersts erhielt. Nach dem Aprilkrieg 1941 betei- 
ligte er sich an der Organisierung des Aufstands vom 13. Juli. Mitglied der Kommunistischen Partei Jugoslawiens ab 1943. Die Partisanen verliehen ihm als Erstem den Rang eines Generalleutnants. Orović fotografierte während des gesamten Krieges und schaffte es, die Negative von Bildern zahlreicher bedeutender Schlachten aufzubewahren, zum Beispiel der Schlacht am Fluss Sutjeska und in Drvar. Mitglied des Antifaschistischen Rats der Volksbefreiung Jugoslawiens.

\section{Pantelić, Radovan}

Fotograf.

Parać, (Ivan) Jakov (geb. 1922)

Geboren in Moseć bei Drniš. Fotograf und Arbeiter, Mitglied des Verbands der Kommunistischen Jugend Jugoslawiens ab 1943 und Angehöriger der Stabseinheit der Ersten Proletarischen Brigade sowie politischer Delegat.

\section{Pašić, Emir}

Mittelschüler und Fotograf der 13. HerzegowinaStoßbrigade der Volksbefreiungsarmee sowie politischer Delegat der Ersten Kompanie des Zweiten Bataillons.

\section{Pavletić, Ivica}

Fotograf.

\section{Pavlić, Drago}

Fotograf.

Pavlin, Miran-Miro (geb. 1921)

Zu Beginn des Zweiten Weltkriegs fotografierte er im besetzten Ljubljana und betrieb heimlich ein Fotostudio in der Gregorič-Straße. Einer der wichtigsten Chronisten im okkupierten Ljubljana.

\section{Pavlović, Živan}

Geboren in Kikinda in Serbien. Er fiel bei Pleternica, wo er auch begraben wurde.
Perajica, (Ivan) Antonio (1915-1992)

Geboren in Čvrljevo bei Drniš. Vor dem Krieg war er als Fotograf tätig und eröffnete bereits 1934 sein eigenes Atelier. Bei Kriegsbeginn arbeitete er für die Cinecittá in Italien. Nach der Kapitulation Italiens gelangte er mit Mitgliedern der GaribaldiBrigade nach Dalmatien. Ab September 1943 Zugführer im Dritten Bataillon der Ersten Proletarischen Brigade, Fotograf und Filmreporter sowie ab 1944 Mitglied der Kommunistischen Partei Jugoslawiens.

Petek, Jože (1912-1945)

Einer der talentiertesten slowenischen Fotografen, der 1943 der Partisanenbewegung beitrat. Er nahm den Feldzug der 14. Division in Kroatien und in der Steiermark auf und schuf Fotografien von besonderem dokumentarischem Wert. Nachdem er verraten wurde, erschoss man ihn am 3. Januar 1945.

\section{Peter, Fajfar}

Fotograf im Neunten Korps.

Petrović, (Jakša) Batrić (geb. 1915)

Aus der Siedlung Klopot bei Podgorica stammend. In der Ersten Proletarischen Brigade ab September 1944. Mitglied des Ersten Bataillons und Fotograf der Brigade.

\section{Pfeifer, Marjan (1910-1992)}

Geboren in Ljubljana. Er besuchte die Grafikschule in Wien und erlernte das Fotografenhandwerk in Graz. In der Partisanenbewegung wirkte er als Fotograf und nahm die Befreiung der Stadt Maribor auf. Vor dem Krieg war er im Verlag Jugoslovanska tiskarna in Ljubljana beschäftigt. Sein Bild Na juriš (Sturmangriff) gilt vielen als eine der besten Reportagefotografien der Partisanen.

Pipenbacher, Gojko (geb. 1902)

Polyglotter und Präsident des Fotoklubs Ljubljana in der Vorkriegszeit. Er schloss sich 1943 der Partisanenbewegung an, organisierte den Fotodienst 
in der 18. Division und war in der Folge als Fotograf in der Agitprop-Abteilung des Siebten Korps aktiv.

Pirnat, Ivan (geb. 1901)

Amateurfotograf, später Redakteur der Zeitschrift Polet und Mitglied der Sektion Slowenischen Volksbefreiungsrates in Črnomelj.

\section{Plavšić, Nikola}

Leiter der Fotosektion des Oberkommandos der Volksbefreiungsarmee und des Landrats für die Vojvodina, zu dem er am 26. Dezember 1944 ernannt wurde.

\section{Pocajt, Marjan}

Mitglied der Foto- und Filmsektion des Siebten Korps.

Popov, Petar (geb. 1895)

Am 5. Mai 1941 wurde er von den Ustascha verhaftet und an einen unbekannten Ort verschleppt.

\section{Popović, Nikola}

Fotograf der 32. Division.

Popović, Slobodan-Bakula (1923-1941)

Während der Užice-Republik wirkte er als Fotograf und Sekretär des Bezirkskomitees des Verbands der Kommunistischen Jugend Jugoslawiens. Als Kämpfer der Ersten Požega-Kompanie fiel er 1941 bei feindlichen Fliegerangriffen im serbischen Požega.

Potočnjak, Vlado (1924-1997)

Maler und Mitglied des Fotodienstes des Regionalen Volksbefreiungsrats für Slawonien.

Povh, Dušan (1921-2000)

Fotografierte die Auswirkungen der italienischen Okkupation seines Geburtsorts Novo Mesto und arbeitete im Atelier für Zentraltechnik der Kommunistischen Partei Sloweniens.
Poznjak, (Jakov) Ivan (geb. 1926)

Fotograf und ab dem 21. Oktober 1944 Mitglied der Zweiten Dalmatinischen Brigade.

Prešern, Jakob (1888-1975)

Geboren in Begunje in der Oberkrain in Slowenien. Soldat im Ersten Weltkrieg. Während des Zweiten Weltkriegs dokumentierte er die italienische Okkupation von Ljubljana und die Befreiung der Stadt.

\section{Radivojević, Dragić}

Fotograf in der Vierten Serbischen Stoßbrigade. Er fiel bei Lovas.

\section{Rakić, Milivoje}

Fotograf.

\section{Rebić, Marijan}

Fotograf aus Knin.

Ritopečki, Jovan (1923-1989)

Fotograf aus Pančevo. Radiotechniker, Kriegsberichterstatter und Fotograf der Ersten Vojvodina-Brigade.

Roca, Ante (1905-1989)

Geboren in Vodice. Er fotografierte ab 1921 und wurde 1924 Mitglied der Kommunistischen Partei. Ab 1943 war er im Volksbefreiungskrieg in der Stabseinheit der 19. Norddalmatinischen Division aktiv und wirkte später als Fotograf des Antifaschistischen Landesrats der Volksbefreiung Kroatiens in der Abteilung für technische Dienste.

Romac, Paško (1913-1982)

Geboren in der Ortschaft Vukšić in der Nähe von Benkovac. In der Vorkriegszeit Kommunist, der wegen seiner politischen Überzeugungen zu einer mehrjährigen Haftstrafe verurteilt wurde. Im August 1941 gelang es ihm, aus dem Gefängnis in Sremska Mitrovica durch einen gegrabenen Tunnel zu fliehen, woraufhin er sich an der Gründung der Partisaneneinheit in der Fruška Gora beteiligte. Später bestellte man ihn zum Politkommissar der Ersten Proletarischen Brigade. 
Als Mitglied der Politabteilung wurde er am 6. September 1942 mit Parteiarbeit betraut, danach erfolgte die Ernennung zum Politkommissar der Ersten Vojvodina-Brigade. Während des gesamten Krieges fotografierte Romac mit seiner eigenen Kamera.

\section{Romanič, Peter}

Mitglied der Fotosektion des Rats der Volksbefreiung Sloweniens, der für Aufnahmen aus dem politischen Leben zuständig war.

Ruljančić, Jure (1913-1998)

Mitglied der Fotosektion des Regionalen Volksbefreiungsrats für Dalmatien.

\section{Šaković, Ismet (1920-1945)}

Fotograf aus Gračanica, der sein Handwerk in Doboj erlernt hatte. Mit fünfzehn Jahren eröffnete er ein eigenes Fotogeschäft. Am 13. März 1945 starb er auf dem Berg Romanija bei Kämpfen gegen die deutschen Besatzer.

Savić, Branko (1915-2009)

Geboren in Bjelovar. In Berlin absolvierte er einen Ausbildungskurs zum Fotografen und kehrte nach der Machtergreifung der Nationalsozialisten nach Belgrad zurück. Gegen Ende des Kriegs fotografierte er für die Nachrichtenagentur TANJUG.

Sege, Nikola (geb. 1909)

Geboren in Vrbas. Er arbeitete als Fotograf in Osijek und gehörte ab 1943 der Volksbefreiungsarmee an.

Šelhaus, Edi (1919-2011)

Aufgrund der Verfolgung durch die Faschisten zog die Familie Šelhaus nach einigen Jahren in Triest nach Zagreb, wo sein Vater ein Fotoatelier eröffnete, während seine Mutter in Škofja Loka arbeitete. 1943 erhielt er einen Einberufungsbefehl der deutschen Wehrmacht, doch schloss er sich der Partisaneneinheit aus Škofja Loka an. Im Sommer 1944 wurde er schwer ver- wundet. Während er auf dem Partisanenflugplatz Podzemelj auf den Transport nach Bari wartete, kam er mit dem Agitprop-Offizier France Brenk in Kontakt, der zu jener Zeit zusammen mit Franjo Veselko den Fotodienst der slowenischen Partisanen organisierte.

Ševkan, (Ibrahim) Revro (1914-1944)

Geboren in Mostar, Bosnien und Herzegowina. Arbeiter und Fotograf, der sich 1942 der Volksbefreiungsarmee anschloss. Er fiel 1944 bei Gornji Vakuf.

Šibelja, Anton (1914-1945)

Werftarbeiter, Mitglied der Bewegung TriestIstrien-Gorica-Rijeka (TIGR) und der Kommunistischen Partei Italiens ab 1936. Organisator der ersten militärischen Aktionen in der Gegend von Triest. 1942 folgte er den Partisanen, bei denen er in der technischen Werkstatt tätig war und überdies als Untergrundkämpfer häufig in das feindliche Territorium reiste. Gegen Kriegsende Leiter der Werkstatt des Neunten Slowenischen Korps. Einer der Erfinder der »Partisanenkanone«, die aus italienischen Mörserrohren mit einem Durchmesser von $81 \mathrm{~cm}$ sowie verschiedenen Waffen- und Fahrzeugteilen zusammengesetzt war. Darüber hinaus fotografierte er während des gesamten Krieges.

\section{Šimunov, Vili-Barba}

Einer der ersten Fotografen des Oberkommandos und der Nachrichtenagentur TANJUG. Er fiel 1944 bei den Kämpfen um Drvar.

Škodlar, Čoro (1902-1966)

Maler, Fotograf und Publizist. Er trat 1943 der Partisanenbewegung bei, in der er für die Beschaffung von Fotomaterial zuständig war. Er war einer der wenigen Fotografen, die Farbaufnahmen gefertigt haben. 
Skrigin, Žorž (1910-1997)

Geboren in Odessa. Von Beruf Balletttänzer im

Kroatischen Nationaltheater und vor dem Krieg

Mitglied des Fotoklubs Zagreb. Skrigin, der in Bel-

grad verstarb, war einer der aktivsten Partisanen-

fotografen und hinterließ eine umfängliche

Fotosammlung.

\section{Sladić, Vjekoslav}

Geboren in Laslavić. Berufsfotograf und Mitglied des Bezirkskomitees der Kommunistischen Partei Kroatiens in Karlovac.

Smolej, Slavko (1909-1961)

Geboren in Jesenice. Wegen seiner Mitwirkung an der Befreiungsfront wurde er am 20. Januar 1945 verhaftet. Fotografierte und entwickelte Filme für die Partisaneneinheiten aus Jesenice und Bohinj.

Sočan, Jule (geb. 1909)

Ab dem Kriegsausbruch im Jahr 1941 illegal tätiger Fotograf in Ljubljana, der für die TomšićBrigade Aufnahmen fertigte.

Solkan, Gorica (gest. 1945)

Fotografin in der Volksbefreiungsarmee ab dem 23. Oktober 1944. Sie fiel am 17. Januar 1945 in der Nähe des Dorfes Sotin in Kroatien.

\section{Spasić, Levča}

Fotograf.

\section{Spasić, Stojan}

Fotograf des Ersten Bataillons und Zeitungsreporter.

\section{Štajer, Božidar}

Fotograf.

\section{Stajer, Jože}

Illegal tätiger Fotograf
Starčević, (Nikola) Blagoje (1927-1943)

Starčević arbeitete im Geschäft Foto Drago des Fotografen Drago Jovanović in Tuzla. Im Alter von 16 Jahren schloss er sich 1943 der neu formierten 18. Kroatischen Brigade an. Er fiel bei Kämpfen gegen Einheiten der deutschen Wehrmacht.

Stevanović, (Milutin) Milivoj (geb. 1903)

Geboren in Ivoševci bei Knin in Kroatien. Ab dem 1. Dezember 1944 im Volksbefreiungskrieg und als Fotograf in der Unteroffizierschule für Luftfahrt aktiv.

\section{Štok, Milan}

Leiter der Fotosektion des Siebten Korps und Autor des Fotografski priručnik (Handbuch der Fotografie).

\section{Šubek, Stjepan}

Fotograf.

\section{Tačković, Mate}

Im April 1945 fotografierte er den Einzug der Verbände der Volksbefreiungsarmee und der Widerstandsbewegung Jugoslawiens in Stara Gradiška. Fotograf der 28. Slawonischen Stoßdivision und des Fotodienstes des Regionalen Volksbefreiungsrats für Slawonien.

\section{Tadić, Nedeljko}

Leiter der Fotosektion der 13. Herzegowina-Stoßbrigade.

Tavčar, Ivan (1889-1966)

Fotograf und Alpinist.

\section{Trobec, Ivan}

Fotograf.

\section{Trobec, Mirko}

Fotograf. 
Vajner, Slaviša, gen. Čiča (1903-1942)

Geboren in Novi Vinodolski. Abitur in Koprivnica und Studium in Zagreb. Im Romanija-Gebirge organisierte er die erste Partisanenkompanie. Mitglied des Hauptstabs des Rats der Volksbefreiung von Bosnien und Herzegowina. Er starb in der Nähe von Han Pijesak durch Selbstmord, um nicht in die Hände der deutschen Wehrmachtssoldaten zu fallen. Vajner trug die ganze Zeit seinen Fotoapparat mit sich.

\section{Vavpotič, Rudi}

Geboren 1919 in Maribor. Mitglied der Fotosektion des Hauptstabs für Slowenien in Vojna Vas bei Črnomelj.

Veselko, Franjo (geb. 1905)

Mitglied der Fotosektion des Hauptstabs für Slowenien und anschließend Leiter der Fotosektion des Rats der Volksbefreiung Sloweniens. Ihm war die Entdeckung mehrerer Tausend deutscher Fotografien in der Redaktion der Zeitschrift Piccolo zu verdanken, unter denen sich Aufnahmen des »Unternehmens Rösselsprung (Angriff auf Drvar) befanden.

\section{Vičić, Emil}

Fotograf, Designer und Architekt. Nach dem Krieg Organisator der Kunstausstellung im Technischen Museum in Zagreb.

Vidin, Vlatko (geb. 1923)

Fotograf und Partisanenkämpfer.

Vidmar, Drago (1901-1982)

Maler. Er studierte in Zagreb und lebte später in Paris. Fotografierte während des Zweiten Weltkriegs und wurde 1944 zum organisatorischen Sekretär des Slowenischen Nationaltheaters berufen.

Vidmar, Nande (1899-1981)

Bruder von Drago Vidmar. Maler, der während des Zweiten Weltkriegs fotografierte. Mitglied der Befreiungsfront ab 1941 und Mitglied der Kommunistischen Partei Jugoslawiens ab 1942. Er hielt die Bewegungen der Partisaneneinheiten und das Begräbnis des Partisanenkommandanten Franc Rozman-Stane fest. Später Mitglied der Fotosektion des Rats der Volksbefreiung Sloweniens und Aquarellmaler.

Viršek, Stane (geb. 1915)

Seine ersten Fotografien nahm er mit der Kamera von Mirko Bračić in der 14. Division auf. In der Folge gehörte er der Ljubljana-Brigade an, in der er bei den Kämpfen gegen den Feind schwer verwundet wurde. Mitglied der Fotosektion des Rats der Volksbefreiung Sloweniens.

\section{Vivoda, Joakim}

Fotograf.

Vujnić, (Mile) Stevo (geb. 1924)

Fotograf aus der Ortschaft Kričke bei Drniš in Kroatien. ab dem 7. Juli 1942 in der Ersten Dalmatinischen Brigade und ab Juli 1943 in der Zweiten Dalmatinischen Brigade. Stellvertreter des Politkommissars des Bataillons.

\section{Vujošević, Jovan}

Fotograf.

Zafron, Frane (geb. 1916)

Dalmatinischer Fotograf, geboren in Vodice. Am 10. März 1943 von den Italienern gefangen genommen. Nach einem Monat in Haft schloss er sich den Partisanen an und kämpfte in der Norddalmatinischen Division.

Zagoričnik, Valerija (geb. 1926)

Geboren in Celje. Fotografin des Hauptstabs der Volksbefreiungsarmee und der Widerstandsbewegung der Vojvodina.

Zalar, Slavko (1921-1987)

Fotograf des Zweiten Bataillons der Ersten Dalmatinischen Brigade, der auf der Insel Vis Aufnahmen fertigte. Später Fotograf des Vierten Bataillons der Ersten Dalmatinischen Brigade und Mitglied der Fotosektion des Regionalen Volksbefreiungsrats für Dalmatien. 


\section{Živković, Borislav}

Fotolaborant und junger Zugführer der 42. Fliegerdivision.

Živković, (Milutin) Djordje (1923-1944)

Amateurfotograf und Landwirt. Ab 1943 Mitglied der Kommunistischen Jugend Jugoslawiens. Er gehörte dem Ersten Bataillon der Volksbefreiungsarmee Serbiens und später der ŠumadijaBrigade an. Am 4. Dezember 1943 wurde er in Prijepolje schwer verwundet, gefangen genommen und am 3. April 1944 im Belgrader Konzentrationslager Sajmište hingerichtet.

\section{Žorž, Bogomir}

Fotograf der Vierten Zone.

Zupančić, Maksimilijan-Milijan (1911-1968) Berufsfotograf, der 1943 den Partisanen folgte. Er organisierte den Fotodienst in Novo Mesto und war anschließend zusammen mit anderen Fotografen an der Gründung des slowenischen Fotodienstes der Partisanen in Črnomelj beteiligt. Große Verdienste erwarb er durch die Beschaffung von Fotomaterialien und die Verbesserung der Qualität der Partisanenfotografie. 